\title{
Postmenopausal Breast Cancer Risk and Cumulative Number of Menstrual Cycles
}

\author{
Mariana Chavez-MacGregor, ${ }^{1,2}$ Sjoerd G. Elias, ${ }^{1}$ N. Charlotte Onland-Moret, ${ }^{1}$ \\ Yvonne T. van der Schouw, ${ }^{1}$ Carla H. Van Gils, ${ }^{1}$ Evelyn Monninkhof, ${ }^{1}$ \\ Diederick E. Grobbee, ${ }^{1}$ and Petra H.M. Peeters ${ }^{1}$
}

${ }^{1}$ Julius Center for Health Sciences and Primary care, University Medical Center, Utrecht, the Netherlands and

${ }^{2}$ Netherlands Institute for Health Sciences, Rotterdam, the Netherlands

\begin{abstract}
Objective: To explore whether the lifetime cumulative number of menstrual cycles, as an index for total exposure to endogenous estrogens, and the number of menstrual cycles until a first full-term pregnancy (FFTP), are associated with breast cancer risk in postmenopausal women.

Methods: Population-based study with data from the Prospect-European Prospective Investigation into Cancer and Nutrition study. Naturally menopausal participants were eligible $(n=6,718)$. The cumulative number of menstrual cycles was computed in $6,031(90 \%)$ women. We calculated the number of cycles until FFTP among parous participants. The number of menstrual cycles was impossible to compute in women who reported to be always irregular; therefore, we added the "always irregular" category in the analysis. During the 46,746 person-years of follow-up, 168 breast cancer cases were identified. Cox regression models were
\end{abstract}

used and adjustments were made to account for potential confounders.

Results: Even when our data does not show a clear linear gradient, we observed an increased breast cancer risk in women with a higher number of cumulative menstrual cycles in their lifetime. Using $\leq 415$ cycles as reference, the hazard ratio for the irregular group, 416-453, 454-490, and $\geq 491$ cycles was $1.11(.56,2.19), 1.88(1.14,3.12), 1.74$ (1.05, $2.87)$, and $1.80(1.09,2.96)$, respectively. Although not statistically significant, and of less magnitude, the risk estimates for the number of cycles before FFTP showed the same tendency.

Conclusion: Among women who underwent natural menopause, a higher number of menstrual cycles in lifetime, reflecting a longer exposure to endogenous estrogens, is associated with an increased breast cancer risk. (Cancer Epidemiol Biomarkers Prev 2005;14(4):799-804)

\section{Introduction}

Although breast cancer is the most common cancer in women worldwide, its causes are not fully understood. Epidemiologic studies show that hormonal factors play a key role. However, the biological mechanisms and possible interaction among risk factors is not yet clearly sufficiently unraveled.

It has been well established that reproductive characteristics are important risk factors for breast cancer (1). Both young age at menarche and late menopause increase breast cancer risk. This could be explained by an increased number of ovulatory cycles and thus exposure to high estrogen levels for a longer period (2). Studies that considered both age at menarche and at menopause have found an increase in breast cancer risk with longer lifetime menstrual activity (3-5).

Childbearing and young age at first full-term pregnancy (FFTP) protect against breast cancer and additional pregnancies further reduce the risk. It has been hypothesized that the overall effect of parity is due to a protective effect of young age at FFTP (6), a likely explanation is that as a result of the action of the hormones during pregnancy, breast epithelial cells undergone differentiation, making them less vulnerable to the effect of carcinogenesis (7). Therefore, according to some

Received 6/21/04; revised 12/9/04; accepted 12/28/04.

Grant support: European Commission-Europe Against Cancer WHO AEP/90/05, Dutch Ministry of Health and Dutch Prevention Funds, and National Council for Science and Technology in Mexico university postgraduate education merit-based academic CONACYT scholarship (M. Chavez-MacGregor).

The costs of publication of this article were defrayed in part by the payment of page charges. This article must therefore be hereby marked advertisement in accordance with 18 U.S.C. Section 1734 solely to indicate this fact.

Requests for reprints: Petra H.M. Peeters, Julius Center for Health Sciences and Primary Care University Medical Center Utrecht, Room STR 6.1.31, P.O. Box 85060, 3508 BA Utrecht, the Netherlands. Phone: 31-30-250-9363, ext. 9384; Fax: 31-30-250-5485. E-mail: P.H.M.Peeters@umcutrecht.nl

Copyright (C) 2005 American Association for Cancer Research. authors, the period between menarche and FFTP may be important for breast cancer etiology $(6,8)$. Some studies have shown an increased risk for the development of breast cancer during and immediately after pregnancy, which has been attributed to the exposure to high levels of estrogen and progesterone. However, after a postpartum period of 10 to 15 years, long-term protection is observed (9).

Breast-feeding has long been hypothesized to protect against breast cancer. Lactation induces final differentiation of the terminal duct epithelium, which makes it relatively insensitive to hormonal stimulation (10). Breast-feeding delays the return of ovulation after pregnancy and is associated with a different hormonal milieu (e.g., increased prolactin) which diminishes the breast's exposure to estrogens (11).

The "lifetime cumulative number of menstrual cycles" may be used as an index that summarizes all the previously mentioned reproductive risk factors. It takes into account determinants that might be relevant when considering the breast's exposure to hormones, such as age at which menstrual cycles became regular, regularity pattern, pregnancies, oral contraceptive (OC) use, and lactation. Although one previous study has shown that women with a larger lifetime cumulative number of menstrual cycles are at increased risk for breast cancer, this observation has thus far not been confirmed (12).

We studied the relation between reproductive characteristics, summarized as the lifetime cumulative number of menstrual cycles and breast cancer risk in naturally menopausal women. Because of the postulated importance of the period between menarche and the FFTP in breast cancer etiology, the number of cycles within this period was also considered in parous participants. The aim was to determine whether the lifetime cumulative number of menstrual cycles and the "number of menstrual cycles until FFTP" are associated with breast cancer risk. 


\section{Materials and Methods}

The Prospect-European Prospective Investigation into Cancer and Nutrition study is one of the two Dutch cohorts participating in the European Prospective Investigation into Cancer and Nutrition, a multicenter prospective study carried out in 23 centers from 10 European countries (13).

A detailed description of the study population and data collection of the Prospect-European Prospective Investigation into Cancer and Nutrition study has been published elsewhere (14). Briefly, 17,357 women ages 49 to 70 years, residing in Utrecht and its surroundings were recruited through a regional, population-based, breast cancer-screening program between 1993 and 1997. Baseline characteristics were collected based on two self-administered questionnaires (regarding lifestyle and nutrition). The lifestyle questionnaire contains information on demographic characteristics, family history, past and current morbidity, reproductive history (gynecologic and obstetric), smoking habits, and physical activity. The nutrition questionnaire is aimed at capturing the habitual diet during the year preceding enrollment. In addition, pulse rate, blood pressure, and anthropometric measurements (height, weight, and waist and hip circumference) were taken. Blood (30 $\mathrm{mL}$ ) was drawn and stored at $-196^{\circ} \mathrm{C}$ (14). All women signed a written informed consent; the Institutional Review Board of the University Medical Center Utrecht approved the study.

From all postmenopausal Prospect-European Prospective Investigation into Cancer and Nutrition participants $(12,817)$, only those who underwent natural menopause were eligible for the present study $(n=6,718)$ because only in them we had reliable information about ovarian activity not masked by the absence of menstrual cycles due to hysterectomy. Natural menopause was defined as the complete cessation of menstrual bleeding for 12 months preceding enrollment due to natural causes (according to self-report). Women with previous history of breast cancer were excluded $(n=121)$ as well as 566 participants with incomplete information to compute the lifetime cumulative number of menstrual cycles. Together, there were 6,031 (90\%) eligible participants.

Cumulative Number of Menstrual Cycles. The lifetime cumulative number of menstrual cycles was computed from age at first menstrual period to age at menopause. For parous women, the number of menstrual cycles before FFTP was also estimated. Ages at menarche, at menopause, and at FFTP were recorded as continuous variables. Age at regularization of cycles was computed according to the time between menarche and the establishment of regular periods (immediately after menarche, after 1, 2, 3, 5, or 10 years, after FFTP or never regular). The regularity pattern was defined categorically as 24-, 26-, 28-, 30-, and 32-day cycles, or always irregular. Information about $\mathrm{OC}$ use included age at start, number of years of use, and age at last use. Data about number of and age at each pregnancy, stillbirth, miscarriage and abortion, if any, were available, as well as the number of weeks of breastfeeding for each pregnancy.

We took all previously mentioned variables into account when computing the lifetime cumulative number of menstrual cycles, considering that each pregnancy resulted in an absence of cycles for a period of 36 weeks, a stillbirth and absence for 28 , and any miscarriage or abortion and absence of cycles for 12 weeks. We assumed that lactation results in a 6-week absence of cycles (15). Menstrual cycles due to OC use were computed as a 28-day duration (see Appendix 1).

Considering that irregular cycles are a combination of long and short menstrual periods, the number of irregular cycles was computed according to each participant's regularity pattern; the total number of irregular years was recorded to make further adjustments. However, the number of menstrual cycles was impossible to compute in those participants who reported always having irregular menstrual cycles or never having regular periods; therefore, we added the "always irregular" category as such in the analysis.

The cohort data were linked to the databases of the municipal registries to ascertain the vital status of all participants and to obtain information about subjects who moved from the region or emigrated abroad. The end point for the study was the diagnosis of primary breast cancer. Until July 2003, 168 cases were identified through record linkage with the Comprehensive Cancer Center Middle Netherlands.

For participants with breast cancer, follow-up ended at the date of diagnosis, for women who left the region or country, the censoring date was the date of emigration, for those who died, follow-up was censored at the date of death. For all others, the censoring date was July 1, 2003.

Data Analysis. The lifetime cumulative number of menstrual cycles and the number of menstrual cycles before FFTP were analyzed as categorical variables (irregular, 25th, 50th, and 75th percentiles). Cox proportional hazard analysis was carried out using follow-up as time scale; the lowest category of number of menstrual cycles was used as reference. Crude and adjusted hazard ratios (HR) for breast cancer are presented, together with $95 \%$ confidence intervals (95\% CI). We adjusted for potential confounding variables, including age at enrollment, body mass index at examination as a continuous variable, $>1$ year of irregular cycles $(y / n)$, mother or sister with history of breast cancer $(\mathrm{y} / \mathrm{n})$, use of hormone replacement therapy ever $(y / n)$, ever married $(y / n)$, fertility consult ever $(y / n)$, and education level. For analyses of lifetime cumulative number of menstrual cycles, the regression variable for age at first childbirth was centered around its mean and multiplied by parity $(\mathrm{y} / \mathrm{n})$, making it possible to include both potential confounders simultaneously into the model $(16,17)$. Further adjustment for smoking habits, ever OC use, and number of years of OC use did not materially changed the results; thus, these variables were not included in the final model.

Trend tests were done by introducing the lifetime cumulative number of menstrual cycles or the number of menstrual cycles before FFTP categories as numerical variables and testing the significance of the models' linearity (Wald's test). Because there is no clear natural hierarchy, the always irregular category was not included when testing for trends. The SPSS statistical package for WINDOWS, version 11.5, was used for all statistical analyses, and all tests were two sided.

\section{Results}

Mean age of this cohort of postmenopausal women was 59.5 years old at baseline, with a mean age at menarche of 13.5 years and at menopause of 49.5 years. The women were followed for a total of 46,746 person-years, with a median follow-up of 7.9 years. Among the 6,031 participants eligible for this study, 168 $(2.8 \%)$ were newly diagnosed with breast cancer, $206(3.42 \%)$ were diagnosed with other types of cancer, $240 \quad(3.98 \%)$ participants died, and $137(2.27 \%)$ emigrated or were lost to follow-up. A total of 5,654 (87.55\%) participants were alive and free from cancer at the end of follow-up (July 1, 2003).

Table 1 presents general characteristics of the ProspectEuropean Prospective Investigation into Cancer and Nutrition participants included in this study, and Table 2 shows some characteristics related to menstrual and reproductive function, indicated according to the lifetime cumulative number of menstrual cycles category. The participants who had the highest lifetime number of menstrual cycles tended to have a higher educational level, a younger age at menarche, a later menopause, an older age at FFTP, a smaller number of live 
born children, and shorter menstrual cycles compared with the participants in the other categories. Furthermore, women with the highest number of menstrual cycles tended to be nulliparous more often and have more frequently breast cancer in their family.

The median number of lifetime menstrual cycles for the study population, after excluding those participants in the always irregular category (in whom the number of menstrual cycles was impossible to compute) was 456.7 cycles, corresponding to 35.1 years of menstrual activity if considering an average of 13 cycles per year. Table 3 presents the crude and adjusted risk estimates between the lifetime cumulative number of menstrual cycles and breast cancer. Compared with participants with fewer than 415 cycles (31.9 years of menstrual activity), the fully adjusted HR of breast cancer for the always irregular participants, the women with a lifetime number of menstrual cycles between 416 and 453 (equivalent to 32-34.8 years of menstrual activity), the participants with 454 to 490 (corresponding to 34.9-37.7 years of menstrual activity), and those with $>491$ menstrual cycles $(>37.8$ years of menstrual activity) was 1.11 (95\% CI, 0.56-2.19), 1.88 (95\% CI, 1.14-3.12), $1.74(95 \% \mathrm{CI}, 1.05-2.87)$, and 1.80 (95\% CI, 1.09-2.96), respectively $\left(P_{\text {trend }}=0.053\right)$. Inclusion of the reproductive terms parity and age at first childbirth (model e) did not change the effects of number of menstrual cycles (models c and d).

The median number of menstrual cycles before FFTP for the parous participants, also excluding the women in the always irregular category, was $146.8 \quad(\sim 11.2$ years of menstrual activity). Table 4 shows the relationship between the number of cycles before FFTP and breast cancer risk computed for the 5,130 parous women in the study. Using as a reference the category $\leq 118$ cycles (corresponding to 9 years of menstrual activity), the fully adjusted HR of breast cancer for the always irregular group, for those participants with 119 to 147 cycles (corresponding to 9.1-11.3 years of menstrual activity), those with 148 to 185 cycles (equivalent to 11.414 .2 years of menstrual activity), and the participants with $>186$ cycles before FFTP (equivalent to $>14.3$ years of menstrual activity), was 0.72 (95\% CI .35-1.46), 1.21 (95\% CI .75-1.96), 1.14 (95\% CI .66-1.95), and $1.27\left(95 \%\right.$ CI .77-2.08), respectively $\left(P_{\text {trend }}=0.407\right)$.

\section{Discussion}

The results of the present study show that women with a larger lifetime cumulative number of menstrual cycles have a significant higher risk of breast cancer. Although not significant and of lesser magnitude, the observed risk estimates for the number of cycles before FFTP show the same behavior. As can be seen in Table 3, the effect of lifetime cycles seems an effect of the lowest category versus the other three, without a clear linear gradient in risk. Although the $P_{\text {trend }}$ is borderline significant, the data seem to show more of a threshold effect than a linear relationship. However, finding where the exact threshold is for number of cycles is not easy. Even in this population of large size, the number of breast cancer events is too limited to precisely pinpoint where the risk is increasing. If numbers in the future increase, empirical or statistical methods, such as splines, may help in finding the threshold value.

To appreciate these findings, some strengths and limitations of our study need to be addressed. The main advantages of this study are its prospective nature, the large sample size, and the completeness of the risk factors data. The fact that participants were recruited through an existing populationbased breast cancer screening program allowed us to include only disease-free participants at enrollment. Finally, the completeness of the Comprehensive Cancer Center Middle Netherlands provided detailed information about any cancer diagnosis and vital status and lowered the proportion of subjects who were lost to follow-up.

It is important to mention that the data we used for the present study are derived from self-administered questionnaires that were filled in at enrollment, long before the diagnosis of breast cancer was made; thus, the answers could not have been influenced by symptoms or awareness of the disease. However, as we used information about past events in each participant's life to compute the lifetime number of menstrual cycles and the number until FFTP, misclassification may have occurred. However, given the prospective nature of our design, if recall errors exist, they would be nondifferential, causing if anything a dilution of our risk estimates. When computing the number of menstrual cycles we made assumptions about the duration of amenorrhea associated with periods of pregnancy, abortion, miscarriage, stillbirth, and lactation, even if these assumptions could lead to some misclassification of the exposure, it also would be nondifferential.

The patterns of risk associated with reproductive history suggest that prolonged exposure to ovarian hormones increases breast cancer occurrence (18). However, only a few epidemiologic studies have tried to combine different reproductive characteristics (as a proxy for ovarian activity) and breast cancer risk $(4,5,12)$. The methodology in the prospective study by Clavel-Chapelon et al. (12) was similar to ours. However, for the computations of the number of menstrual cycles, they assumed an irregular cycle to be equivalent to one of two cycles and we assumed that an irregular cycle was

Table 1. General characteristics of the study population

\begin{tabular}{|c|c|c|c|c|c|}
\hline & \multicolumn{5}{|c|}{ Lifetime cumulative number of menstrual cycles } \\
\hline & $\begin{array}{l}\leq 415 \\
(n=1,313)\end{array}$ & $\begin{array}{l}416-453 \\
(n=1,203)\end{array}$ & $\begin{array}{l}454-490 \\
(n=1,329)\end{array}$ & $\begin{array}{l}\geq 491 \\
(n=1,388)\end{array}$ & $\begin{array}{l}\text { Always irregular } \\
(n=798)\end{array}$ \\
\hline Age at enrollment ${ }^{*}$ & $59.45(5.87)$ & $58.83(5.51)$ & $59.56(5.19)$ & $60.4(4.9)$ & $59.2(5.6)$ \\
\hline Body mass index ${ }^{*}$ & $25.98(4.23)$ & $25.89(3.98)$ & $26.06(3.95)$ & $26.44(4.1)$ & $26(4.3)$ \\
\hline \multicolumn{6}{|l|}{ Educational level (\%) } \\
\hline Primary education & $401(30.5)$ & 309 (25.7) & $308(23.2)$ & $339(24.4)$ & $217(27.2)$ \\
\hline Lower technical, secondary & $583(44.1)$ & $574(47.7)$ & $623(46.9)$ & $614(44.2)$ & $368(46.1)$ \\
\hline High technical, secondary & $162(12.3)$ & $160(13.3)$ & $176(13.2)$ & $204(14.7)$ & $102(12.8)$ \\
\hline Academic & $167(12.7)$ & $160(13.3)$ & $222(16.7)$ & $231(16.6)$ & $111(13.9)$ \\
\hline Ever married (\%) & $1,257(95.7)$ & $1,144(95.1)$ & $1,241(93.37)$ & $1,221(88.0)$ & $729(91.4)$ \\
\hline \multicolumn{6}{|l|}{ Cigarette smoking habits (\%) } \\
\hline Never smoked & $625(47.6)$ & $561(46.7)$ & $632(47.6)$ & $675(48.6)$ & $350(43.9)$ \\
\hline Past smoker & $382(29.1)$ & $384(31.9)$ & $444(33.4)$ & $478(34.4)$ & $282(35.3)$ \\
\hline Current smoker & $306(23.3)$ & $257(21.4)$ & $253(19.0)$ & $235(16.9)$ & $166(20.8)$ \\
\hline Mother or sister with breast cancer (\%) & $153(11.7)$ & $139(11.6)$ & $182(13.7)$ & $214(15.4)$ & $101(12.7)$ \\
\hline
\end{tabular}

${ }^{*}$ Mean \pm SD. 
Table 2. Characteristics related to menstrual and reproductive function in the study population

\begin{tabular}{|c|c|c|c|c|c|}
\hline & \multicolumn{5}{|c|}{ Lifetime cumulative number of menstrual cycles } \\
\hline & $\begin{array}{l}\leq 415 \\
(n=1,313)\end{array}$ & $\begin{array}{l}416-453 \\
(n=1,203)\end{array}$ & $\begin{array}{l}454-490 \\
(n=1,329)\end{array}$ & $\begin{array}{l}\geq 491 \\
(n=1,388)\end{array}$ & $\begin{array}{l}\text { Always irregular } \\
(n=798)\end{array}$ \\
\hline Age at menarche* & $14.1(1.8)$ & $13.69(1.5)$ & $13.3(1.5)$ & $12.8(2.0)$ & $13.8(1.8)$ \\
\hline \multicolumn{6}{|l|}{ Establishment of regular periods $(\%)^{\dagger}$} \\
\hline Immediately after menarche & 994 (12.4) & $918(76.3)$ & $989(74.4)$ & $1,088(78.4)$ & \\
\hline $1-2$ y after menarche & $243(18.5)$ & $241(20.0)$ & $283(21.3)$ & $247(19.8)$ & \\
\hline 3-4 y after menarche & $31(2.3)$ & $9(0.8)$ & $26(1.9)$ & $21(1.5)$ & \\
\hline First 10 y not regular & $18(1.4)$ & $11(0.9)$ & $13(1.0)$ & $20(1.4)$ & \\
\hline Regular after FFTP & $27(2.1)$ & $24(2.0)$ & $18(1.4)$ & $12(0.9)$ & \\
\hline \multicolumn{6}{|l|}{ Periodicity pattern $(\%)^{\dagger}$} \\
\hline Every $26,25,24 \mathrm{~d}$ or less & $163(12.4)$ & $184(15.3)$ & $289(21.8)$ & $738(53.2)$ & \\
\hline Every 27,28 or $29 \mathrm{~d}$ & $906(69)$ & $862(71.7)$ & $928(69.8)$ & $610(43.9)$ & \\
\hline Every $30,31,32 \mathrm{~d}$ or more & 244 (18.6) & 157 (13.0) & $112(8.4)$ & $40(2.9)$ & \\
\hline Nulliparous (\%) & $114(8.6)$ & $126(10.5)$ & 188 (14.1) & $333(24.0)$ & $140(17.5)$ \\
\hline Age at FFTP ${ }^{*,+}$ & $25.2(3.8)$ & $25.4(3.6)$ & $25.7(3.9)$ & $26.4(4.3)$ & $25.4(4.0)$ \\
\hline Number live born children ${ }^{*}$, & $3.43(1.8)$ & $2.91(1.2)$ & $2.7(1.1)$ & $2.5(1.13)$ & $2.85(1.4)$ \\
\hline \multicolumn{6}{|l|}{ Miscarriage and abortions (\%) } \\
\hline Never pregnant & $99(7.5)$ & $114(9.5)$ & $168(12.6)$ & $314(22.6)$ & $125(15.7)$ \\
\hline None & $804(61.2)$ & 747 (62.1) & 849 (63.9) & $820(59.1)$ & $476(59.6)$ \\
\hline 1 & $274(20.9)$ & 247 (20.5) & $222(16.7)$ & 185 (13.3) & 141 (17.7) \\
\hline$\geq 2$ & $136(10.4)$ & $95(7.9)$ & $90(6.8)$ & $69(5.0)$ & $56(7.0)$ \\
\hline \multicolumn{6}{|l|}{ Stillbirth (\%) } \\
\hline Never pregnant & $99(7.5)$ & $114(9.5)$ & $168(12.6)$ & 314 (22.6) & 125 (15.7) \\
\hline None & $1,155(88)$ & $1,044(86.8)$ & $1,118(84.1)$ & $1,041(75.0)$ & $649(81.3)$ \\
\hline$\geq 1$ & $59(4.5)$ & $45(3.7)$ & $43(3.3)$ & $33(2.4)$ & $24(3.0)$ \\
\hline Fertility consult ever (\%) & $128(9.7)$ & $100(8.3)$ & $153(11.5)$ & $171(12.3)$ & $118(14.8)$ \\
\hline Age at menopause* & $45.3(4.4)$ & $49.2(2.5)$ & $50.9(2.3)$ & $52.7(2.7)$ & $48.7(5.5)$ \\
\hline Oral contraceptive use (\%) & $619(47.1)$ & $624(51.9)$ & $683(51.4)$ & $591(42.6)$ & $410(51.4)$ \\
\hline OC use $(y)^{\S}$ & $6.1(4.6)$ & $6.5(4.8)$ & $7.0(5.21)$ & $6.7(5.5)$ & $5.9(5.1)$ \\
\hline HRT use (\%) & $144(11.0)$ & $112(9.3)$ & $100(7.1)$ & $98(7.1)$ & 91 (11.4) \\
\hline HRT use $(y)^{\prime \prime}$ & $3(3.7)$ & $2.2(2.1)$ & $2.5(2.2)$ & $2.6(2.5)$ & $2.7(2.6)$ \\
\hline
\end{tabular}

NOTE: Abbreviation: HRT, hormone replacement therapy.

${ }^{*}$ Mean $\pm \mathrm{SD}$.

†Participants in the irregular category reported to be never regular.

$\ddagger$ Among parous women.

\$Mean \pm SD among OC users.

Mean \pm SD among HRT users.

equivalent to the usual regularity pattern of each participant. We did this, considering the variation of the human menstrual cycle and that irregularity is characterized by a combination of long and short cycles $(19,20)$. In addition, we further adjusted for the absence or presence of irregular periods for $>1$ year. It is important to mention that computing the number of irregular cycles as one of two or as two of one did not materially change our results (data not shown).

The previously mentioned study included participants who underwent natural as well as surgical menopause, whereas ours only included the former. Another important difference is that they included premenopausal participants, whereas we only included postmenopausal women, which allowed us to compute a reliable estimate of the lifetime number of menstrual cycles. Because of smaller numbers in our study and because we computed the number of menstrual cycles in a slightly different way, we chose different cutoff levels than Clavel-Chapelon et al. did in their study to obtain a more stable reference category. However, when using the same cutoff levels $(\leq 403$ as a reference and compared with 404-411, 442-480, 481-520, and $\geq 521$ menstrual cycles), our risk estimates showed a similar

Table 3. HRs and $95 \%$ Cls for breast cancer risk by categories of the lifetime cumulative number of menstrual cycles

\begin{tabular}{|c|c|c|c|c|c|c|}
\hline \multirow[t]{2}{*}{ No. menstrual cycles } & \multirow[t]{2}{*}{ Cancer cases } & \multirow[t]{2}{*}{ Person-years } & \multirow{2}{*}{$\frac{\text { Crude model }}{\text { HR }(95 \% \text { CI })}$} & \multicolumn{3}{|c|}{ Adjusted models } \\
\hline & & & & $\mathrm{HR}^{*}(95 \% \mathrm{CI})$ & $\mathrm{HR}^{\dagger}(95 \% \mathrm{CI})$ & $\mathrm{HR}^{\ddagger}(95 \% \mathrm{CI})$ \\
\hline Always irregular & 15 & 6,203 & $1.03(0.54-1.97)$ & 1.09 & $1.11(0.56-2.19)$ & $1.11(0.56-2.19)$ \\
\hline$\leq 415$ & 24 & 10,213 & $1^{\S}(0.01 .0)$ & $1^{\S}$ & $1^{\S}(0.002 .1)$ & $1^{\S}$ \\
\hline $416-453$ & 41 & 9,273 & $1.88(1.14-3.11)$ & $1.91(1.15-3.16)$ & $1.88(1.14-3.78)$ & $1.89(1.14-3.12)$ \\
\hline $454-490$ & 42 & 10,273 & $1.75(1.06-2.88)$ & $1.74(1.05-2.88)$ & $1.74(1.05-2.87)$ & $1.74(1.05-2.87)$ \\
\hline$\geq 491$ & 46 & 10,760 & $1.82(1.11-2.97)$ & $1.77(1.08-2.91)$ & $1.80(1.09-2.96)$ & $1.80(1.09-2.96)$ \\
\hline$P_{\text {trend }} \|$ & & & 0.043 & 0.065 & 0.053 & 0.053 \\
\hline
\end{tabular}

NOTE: Cox model with follow-up as time scale. $n=6,031$ (168 breast cancer cases).

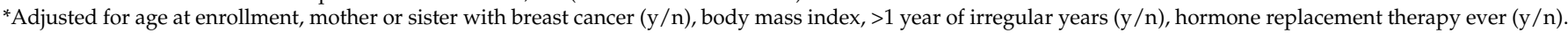

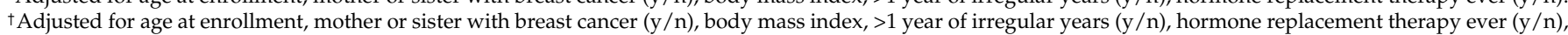
ever married $(\mathrm{y} / \mathrm{n})$, infertility consult ever $(\mathrm{y} / \mathrm{n})$, and educational level.




parity).

§eference category.

\|The irregular category was not included when testing for trend. 
Table 4. HR and $95 \%$ Cls for breast cancer risk by categories of the cumulative number of menstrual cycles before FFTP

\begin{tabular}{|c|c|c|c|c|c|}
\hline \multirow[t]{2}{*}{ No. menstrual cycles } & \multirow[t]{2}{*}{ Cancer cases } & \multirow[t]{2}{*}{ Person-years } & \multirow{2}{*}{$\frac{\text { Crude model }}{\text { HR }(95 \% \text { CI })}$} & \multicolumn{2}{|c|}{ Adjusted models } \\
\hline & & & & $\operatorname{HR}(95 \% \mathrm{CI})^{*}$ & $\operatorname{HR}(95 \% \mathrm{CI})^{\dagger}$ \\
\hline Always irregular & 12 & 5,113 & $0.72(0.37-1.41)$ & $0.71(0.35-1.44)$ & $0.72(0.35-1.46)$ \\
\hline$\leq 118$ & 30 & 9,127 & $1^{\ddagger}$ & $1^{\ddagger}$ & $1^{\ddagger}$ \\
\hline $119-147$ & 39 & 9,565 & $1.25(0.78-2.01)$ & $1.24(0.77-1.99)$ & $1.21(0.75-1.96)$ \\
\hline $148-185$ & 25 & 6,568 & $1.17(0.69-1.99)$ & $1.15(0.67-1.96)$ & $1.14(0.66-1.95)$ \\
\hline$\geq 186$ & 38 & 9,343 & $1.25(0.77-2.01)$ & $1.23(0.76-1.98)$ & $1.27(0.77-2.08)$ \\
\hline$P_{\text {trend }}{ }^{s}$ & & & 0.351 & 0.494 & 0.407 \\
\hline
\end{tabular}

NOTE: Cox model with follow up as time scale. $n=5,130$ (144 breast cancer cases).

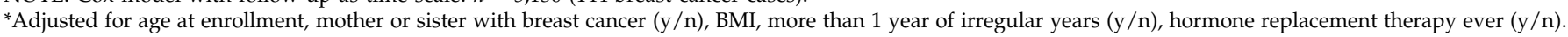

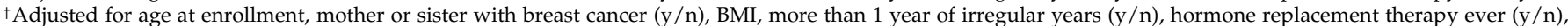
ever married $(\mathrm{y} / \mathrm{n})$, infertility consult ever $(\mathrm{y} / \mathrm{n})$, and educational level.

$\neq$ Reference category.

§The irregular category was not included when testing for trend.

tendency $(1.77,1.35,1.83$, and 1.50$)$ to their results $(0.95,1.19$, 1.20 , and 1.56). Clavel-Chapelon et al. had a larger number of participants, which allowed them to make subgroup analysis among never OC users (12). In our study, an attempt was made to examine subgroups of never OC users, obese participants, and nulliparous women. However, due to the limited number of cases, no reliable estimates could be obtained.

Despite certain differences in methods, and risk estimates of different magnitude, our results are consistent with previous publications addressing this topic $(4,5,12)$ showing that there is an increase in breast cancer risk with an increasing number of menstrual cycles. Collectively, these findings suggest that the number of menstrual cycles during lifetime has a greater risk impact than the number until FFTP. This supports the view that breast's susceptibility to hormones is not of a greater importance during the first reproductive years when the breast epithelial cells are not fully differentiated yet. The duration of exposure to ovarian hormones seems closely related to breast cancer risk, which seems to indicate that not only vulnerable periods are important but also the accumulated time the breasts have been exposed to fluctuating hormone levels.

The hormonal and reproductive factors that have often been related to the risk of breast cancer can be viewed as indicators of certain biological phenomena. Ovarian hormone concentrations are highest during the luteal phase of the menstrual cycle. Women with irregular cycles as well as those with anovulatory cycles spent relatively less of their reproductive years in the luteal phase; thus, they are expected to have a lower risk of breast cancer (18). There is currently no clear evidence of the effect of menstrual irregularity on breast cancer risk, and studies show inconclusive results (21-23), with our and other studies showing no association between irregularity and breast cancer risk $(5,24-27)$.

In summary, the results of this long term prospective study in 6,031 women supports the view that a longer life time exposure to ovulatory hormonal cycles raises the risk of breast cancer. These findings emphasize the relevance of reproductive characteristics in reducing or increasing breast cancer risk and suggest an approach to estimate the full hormonal exposure.

\section{Appendix A}

This is a simple example of a woman with an age at menarche of 12 years, one miscarriage at 23, an age at FFTP of 25, and two children who she breast-feed 10 and 7 weeks. This participant also reported to have regular periods since her menarche with cycles every 30 days, OC use during 5 years starting at age 29, and having menopause at age 51 . Her number of menstrual cycles until her FFTP is 147 and the number of menstrual cycles during her lifetime is 452.8 .

Number of cycles until FFTP: 25 years at FFTP -12 years at menarche $=13$ years.

13 years $\times 52.178$ weeks in 1 year $=678.314$ weeks in 13 years - (12 weeks miscarriage at 23) - (36 weeks first pregnancy) $=630.31$ weeks $=4,861.92$ days.

$4,412.198$ days / $30=147.07$ cycles (30-day duration).

Lifetime number of menstrual cycles: 51 years at menopause -12 years at menarche $=39(-5$ years OC use $=34) ; 34$ years $\times$ 52.178 weeks in 1 year $=1,774.071$ weeks in 34 years $-(12$ weeks of miscarriage $)-(36 \times 2$ weeks of two pregnancies $)-$ (17 weeks total breast-feeding $)-(6 \times 2$ weeks absence of cycles after lactation) $=1,661.071$ weeks $=11,627.5$ days .

$11,627.5$ days $/ 30=387.58$ cycles (30-day duration).

5 years of OC use $\times 365.25=1,461$ days in 5 years, $1,826.25 /$ $28=65.22$ cycles (28-day duration due to OC use), $387.58+$ $65.22=452.8$ menstrual cycles during lifetime.

\section{References}

1. Hulka BS, Moorman PG. Breast cancer: hormones and other risk factors. Maturitas 2001;38:103-13.

2. Key TJ, Verkasalo PK, Banks E. Epidemiology of breast cancer. Lancet Oncol 2001;2:133-40.

3. De Stavola BL, Wang DY, Allen DS, et al. The association of height, weight, menstrual and reproductive events with breast cancer: results from two prospective studies on the island of Guernsey (United Kingdom). Cancer Causes Control 1993;4:331-40.

4. Rautalahti M, Albanes D, Virtamo J, Palmgren J, Haukka J, Heinonen OP. Lifetime menstrual activity: indicator of breast cancer risk. Eur J Epidemiol 1993;9:17-25.

5. Whelan EA, Sandler DP, Root JL, Smith KR, Weinberg CR. Menstrual cycle patterns and risk of breast cancer. Am J Epidemiol 1994;140:1081-90.

6. MacMahon B, Cole P, Lin MT, et al. Age at first birth and cancer of the breast. A summary of an international study. Bull World Health Organ 1970;43:209-21.

7. de Waard F. Endocrine aspects of cancer: an epidemiological approach. J Steroid Biochem Mol Biol 1991;40:15-9.

8. de Waard F. Risk factors for breast cancer at various ages. Eur J Cancer Prev 1998;7:S13-5.

9. Lambe M, Hsieh C, Trichopoulos D, Ekbom A, Pavia M, Adami HO. Transient increase in the risk of breast cancer after giving birth. $\mathrm{N}$ Engl J Med 1994;331:5-9.

10. Russo J, Russo IH. Cellular basis of breast cancer susceptibility. Oncol Res 1999;11:169-78

11. Newcomb PA, Storer BE, Longnecker MP, et al. Lactation and a reduced risk of premenopausal breast cancer. N Engl J Med 1994;330:81-7.

12. Clavel-Chapelon F. Cumulative number of menstrual cycles and breast cancer risk: results from the E3N cohort study of French women. Cancer Causes Control 2002;13:831-8.

13. Riboli E, Hunt KJ, Slimani N, et al. European Prospective Investigation into Cancer and Nutrition (EPIC): study populations and data collection. Public Health Nutr 2002;5:1113-24. 
14. Boker LK, van Noord PA, van der Schouw YT, et al. Prospect-EPIC Utrecht: study design and characteristics of the cohort population. European Prospective Investigation into Cancer and Nutrition. Eur J Epidemiol 2001; 17:1047-53.

15. Cunningham FG, Gant NF, Leveno KJ, Gilstraap LC III, Hauth JC, Wenston KD. Williams obstetrics. 21st ed. USA: McGraw-Hill; 2001.

16. Trichopoulos D, Hsieh CC, Mac Mahon B, Lin TM, Lowe CR, Mirra AP. Age at any birth and breast cancer risk. Int J Cancer 1983;31:701-4.

17. Breslow NE, Day NE. Statistical methods in cancer research. Vol. I. The analysis of case-control studies. IARC Scientific Publications No. 32, Lyon: IARC; 1980

18. Henderson BE, Ross RK, Judd HL, Krailo MD, Pike MC. Do regular ovulatory cycles increase breast cancer risk? Cancer 1985;56:1206-8.

19. Harlow SD, Ephoss SA. Epidemiology of menstruation and its relevance to women's health. Epidemiol Rev 1995;17:265-86.

20. Treolar AE, Boynton RE, Benh BG, Brown BW. Variation of the human menstrual cycle through reproductive life. Int J Fertil 1967;12:77-126.

21. La Vecchia C, Decarli A, Di Pietro S, Franceschi S, Negri E, Parazzini F.
Menstrual cycle patterns and the risk of breast disease. Eur J Cancer Clin Oncol 1985;21:417-22.

22. Salber EJ, Trichopulos D, MacMahon B. Lactation and reproductive histories of breast cancer patients in Boston, 1965-66. J Natl Cancer Inst 1969;43:1013-24.

23. Choi NW, Howe GR, Miller AB, et al. An epidemiologic study of breast cancer. Am J Epidemiol 1978;107:510-21.

24. Titus-Ernstoff L, Longnecker MP, Newcomb PA, et al. Menstrual factors in relation to breast cancer risk. Cancer Epidemiol Biomarkers Prev 1998;7:783-9.

25. Kvale G, Heuch I. Menstrual factors and breast cancer risk. Cancer 1988;62:1625-31.

26. Olsson H, Landin-Olsson M, Gullberg B. Retrospective assessment of menstrual cycle length in patients with breast cancer, in patients with benign breast disease, and in women without breast disease. J Natl Cancer Inst 1983;70:17-20.

27. Parazzini F, La Vecchia C, Negri E, Franceschi S, Tozzi L. Lifelong menstrual pattern and risk of breast cancer. Oncology 1993;50:222-5. 